\title{
Margens cirúrgicas no dermatofibrossarcoma protuberans: relato de caso e revisão da literatura
}

\author{
Surgical margins of dermatofibrosarcoma protuberans: case report and literature review
}

Eduardo R. Z. Câmara ${ }^{1}$, Tricie Kobylko de Toledo², Otávio Alberto Kobylko de Toledoํㅗ Antonio C. Eckhardt ${ }^{1}$, Alessandro A. B. R. Alves ${ }^{1}$, Marcelo S. Araújo ${ }^{1}$, Jadivan L. Oliveira ${ }^{1}$

\begin{abstract}
RESUMO
Introdução: O dermatofibrossarcoma protuberante (DFSP) é um tumor fibrohistiocítico de origem dérmica raro, que acomete a pele, representando $1 \%$ dos sarcomas de partes moles e menos de $0,1 \%$ de todas malignidades. A principal característica deste tipo de tumor é a sua elevada taxa de recidiva local após excisão cirúrgica. Decidir a margem cirúrgica adequada para a ressecção completa é um desafio. Relato de caso: 24 anos, apresenta lesão tumoral vegetante avermelhada no ombro esquerdo, assintomática. Possui história familiar positiva de DFSP em membro inferior. O laudo histopatológico definitivo confirmou Dermatofibrossarcoma protuberans. Discussão: A característica histológica do tumor é a presença de projeções tipo tentáculos de células neoplásicas na periferia que se estendem através do tecido subcutâneo até a fáscia muscular. Certamente, o fator de maior importância para o controle local é a obtenção de margens cirúrgicas livres. A excisão através da cirurgia micrográfica de Mohs é uma ótima opção em regiões onde a excisão ampla não é desejável, como na face. A margem padrão estabelicida em grande parte da literatura é de $3 \mathrm{~cm}$ com ressecção até a fáscia muscular, podendo ser diminuida para $2 \mathrm{~cm}$ em locais em que a excisão ampla prejudique a conformação do local, como na face
\end{abstract}

Palavras chave: Dermatofibrossarcoma protuberante. Neoplasias Cutâneas. Retalho Miocutâneo. Margens Cirúrgicas.

\begin{abstract}
Introduction: Dermatofibrosarcoma protuberans (DFSP) is a rare dermal fibrohistiocytic tumor that affects the skin, accounting for $1 \%$ of soft tissue sarcomas and representing less than $0.1 \%$ of all malignancies. The main characteristic of this type of tumor is a high rate of local recurrence after surgical excision. Deciding the proper surgical margin for complete resection is a challenge. Case report: 24 year-old, shows a reddish vegetative asymptomatic tumor on the left shoulder. She has a positive family history of DFSP in the lower limb. The definitive histopathological report confirmed Dermatofibrossarcoma protuberans. Discussion: The histological feature of the tumor
\end{abstract}

1. Instituto Nacional do Câncer, Rio de Janeiro, Brasil

2. Hospital Universitário Gafree e Guinle
CORRESPONDÊNCIA: Eduardo Rodrigues Zarco da Câmara Instituto Nacional do Câncer Praça da Cruz Vermelha, 23 20230-130 - Rio de Janeiro, Brasil

Recebido em 13/03/2018 Aprovado em 26/06/2018 
is the presence of tentacle type projections of neoplastic cells in the periphery extending through the subcutaneous tissue to the muscular fascia. Of course, the most important factor for local control is the achievement of free surgical margins. Excision through Mohs micrographic surgery is a great option in regions where wide excision is not desirable, as in the face. The standard margin in most pf the literature is $3 \mathrm{~cm}$ resected to the muscular fascia, and it can be reduced to $2 \mathrm{~cm}$ in places where the extensive excision impairs the conformation of the site, as in the face.

Key words: Dermatofibrosarcoma Protuberans. Skin Neoplasms. Myocutaneous Flap. Margins, Surgical.

\section{INTRODUÇÃO}

O dermatofibrossarcoma protuberante (DFSP) é um tumor fibrohistiocítico de origem dérmica, representa $1 \%$ dos sarcomas de partes moles e menos de $0,1 \%$ de todas as malignidades. ${ }^{1,2,3}$ É uma neoplasia maligna rara, que acomete a pele numa incidência aproximada de 0,8 a 4,5 casos por milhão de indivíduos/ano. ${ }^{1,3,4}$ Apresenta crescimento local agressivo, alta taxa de recorrência, mas baixo potencial metastático. ${ }^{5} \mathrm{O}$ sítio de acometimento mais comum é o tronco, seguido das extremidades. Seu crescimento usualmente é lento, e sua agressividade e recorrência frequentemente se limitam ao seu sítio de origem, com rara disseminação linfática ou hematogênica. ${ }^{5,6,7}$ Diagnósticos tardios são comuns por conta de sua aparência benigna e raridade. ${ }^{3,7} \mathrm{O}$ diagnóstico diferencial faz-se com linfomas, sarcoidose, melanoma, metástases cutâneas, quelóides, tumor desmóide, fibrossarcoma, tumores de anexos e dermatofibroma. O dermatofibrossarcoma protuberante deve sempre ser pensado nos casos de recorrência de lesão com diagnóstico histológico de fibroma. ${ }^{2,4}$

A principal característica deste tipo de tumor é a sua elevada taxa de recidiva local após excisão cirúrgica. ${ }^{1,3,6,7}$ Decidir a margem cirúrgica adequada para a ressecção completa é um desafio. Tradicionalmente, o tratamento recomendado para DFSP é a ressecção ampla do tumor, com margem de $3 \mathrm{~cm}$ incluindo a fáscia subjacente, todavia, essa margem é discutida na literatura e muitos advogam o papel da cirurgia micrográfica de Mohs como padrão para o tratamento. ${ }^{2,4,6,7}$

O objetivo desse artigo é relatar um caso de DFSP e discutir sobre as margens ideais para o tratamento dessa neoplasia rara.

\section{RELATO DO CASO}

Paciente feminina, branca, 24 anos, apresenta lesão tumoral vegetante avermelhada no ombro esquerdo, , assintomática, endurecida, de $7 \mathrm{~cm}$, com surgimento há dois anos e crescimento progressivo em seis meses (Figura 1). Possui história familiar positiva de sua mãe portadora de DFSP em membro inferior. Realizada biópsia incisional com laudo histopatológico de DFSP. Microscopicamente, o tumor consiste em células fusiformes atípicas em um estroma fibrótico, na derme e envolvendo o subcutâneo, organizadas em fascículos irregulares, entrelaçados, resultando em padrão estoriforme ou em roda de carroça . A ati-

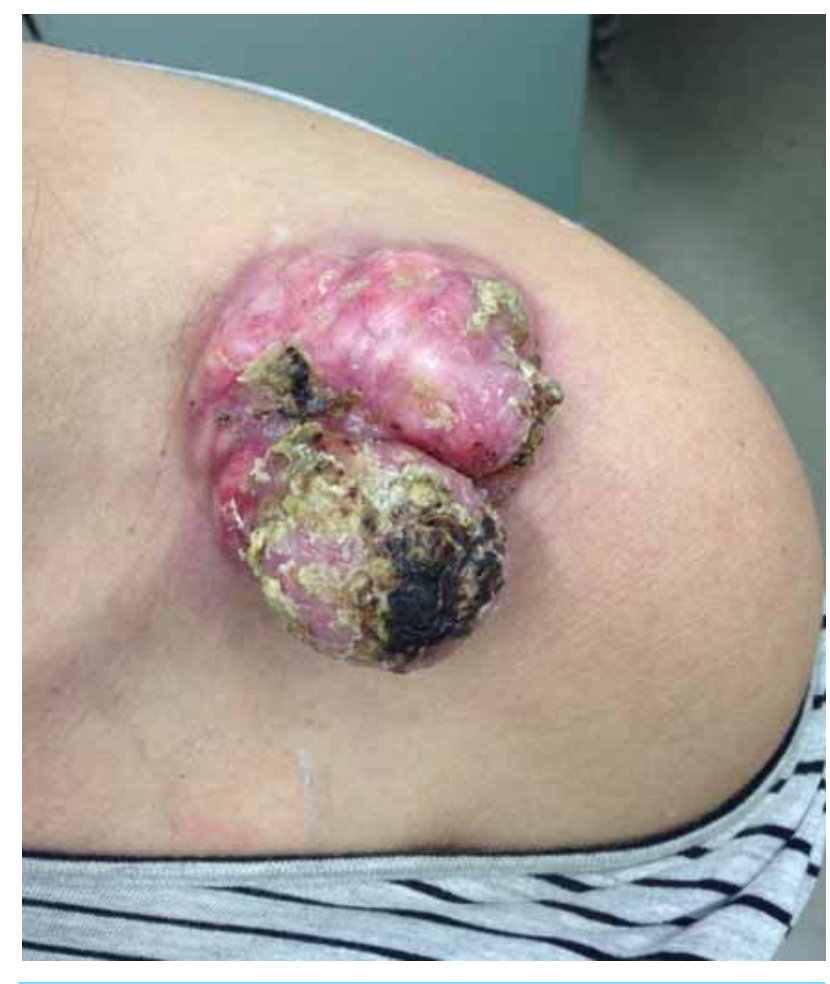

Figura 1: Dermatofibrossarcoma protuberans no ombro esquerdo. 
vidade mitótica é leve e o pleomorfismo nuclear é mínimo, explicando o comportamento do tumor ser geralmente de baixo grau. ${ }^{1,4,7}$ Foi submetido a ressecção ampla da lesão com margem de $3 \mathrm{~cm}$ circunferencial, tendo em vista tamanho da lesão e protocolos da Instiuição, até a fáscia muscular, e recontrução do defeito com retalho miocutâneo trapézio (Figura 2). O laudo histopatológico definitivo confirmou DFSP, com margens livres. A paciente segue em controle ambulatorial há dois anos sem evidência de doença.

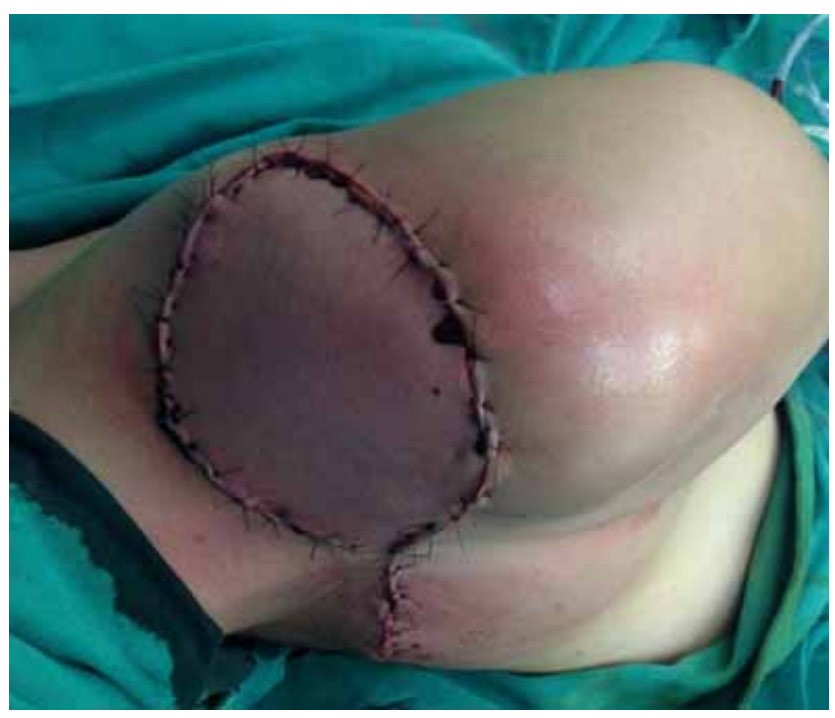

Figura 2: Reconstrução com retalho miocutâneo de trapézio.

\section{DISCUSSÃO}

Dermatofibrossarcoma protuberans é um sarcoma de partes moles raro caracterizado por crescimento local, propensão para envolvimento subclínico e recorrência local. ${ }^{1,2,3,8,9,10}$ Essa neoplasia ocorre mais frequentemente em homens entre $3^{a}$ e $5^{a}$ décadas de vida, no tronco e região proximal das extremidades, mas pode acometer qualquer área da pele. ${ }^{8,11} \mathrm{~A}$ característica histológica do tumor é a presença de projeções tipo tentáculos de células neoplásicas na periferia que se estendem através do tecido subcutâneo até a fáscia muscular. ${ }^{3,4,10}$ Fields et at. recomendam a remoção da fáscia para ressecar completamente as células tumorais de crescimento vertical, visto que a profundidade do tumor interfere na sobrevida livre de doença. 9,10,11 Subtipo histológico, alto índice mitótico, celularidade, tamanho, localização na cabeça e pescoço e lesões recorrentes são fatores associados com maiores taxas de recidiva. ${ }^{12}$ Certamente, o fator de maior importância para o controle local é a obtenção de margens cirúrgicas livres. Quando as ressecções são realizadas com margens inadequadas, a taxa de recidiva local descrita na literatura pode atingir $60 \% .1,5,11$ A ressecção cirúrgica estabelecida como padrão na literatura é de $3 \mathrm{~cm}$ com índice de recorrência em torno de $10 \% .7,8,9 \mathrm{O}$ fechamento primário na maioria dos casos é dificultado, sendo realizada enxertia de pele, uso de retalhos ou cicatrização por segunda intenção. ${ }^{12,13} \mathrm{~A}$ excisão através da cirurgia micrográfica de Mohs é uma ótima opção em regiões onde a excisão ampla não é desejável, como na face. Embora a experiência com a cirurgia de Mohs no tratamento do DFSP seja relativamente limitada, as taxas de recorrência são baixas ( 0 a $6,6 \%$ ), pois permite uma avaliação completa e precisa das margens cirúrgicas do tumor. ${ }^{5,7,11}$

O uso do inibidor seletivo da tirosina-quinase (Imatinibe) vem sendo estudado, demonstrando alguma atividade em pacientes com dermatofibrossarcoma irressecável ou metastático. O Imatinibe funciona como um inibidor do receptor do fator de crescimento derivado de plaquetas, bloqueando a estimulação autócrina desse fator. Estudos clínicos são necessários para determinar se o uso neo-adjuvante do Imatinibe pode reduzir o tamanho do tumor e facilitar a ressecção, assim como, se seu uso adjuvante em combinação com a ressecção do dermatofibrossarcoma diminuiria a taxa de recidiva do tumor. $5,7,10$

A maioria das recorrências é detectada dentro de três anos após a excisão primária, mas estas podem aparecer após 10 anos da operação. Devido à alta taxa de recorrência local, são recomendáveis reavaliações a cada seis meses, com realização de biópsias de áreas suspeitas. O desenvolvimento de metástases é raro, ocorrendo em menos de $4 \%$ dos pacientes, e normalmente é precedido por múltiplas recidivas após excisão inicial inadequada. O pulmão é o principal local de acometimento metastático. Entretanto, lesões cerebrais e ósseas foram descritas. A margen cirúrgica livre é o principal fator de controle local do DFSP. $2,7,8$ e é um fator que mostrou impacto na sobrevida, portanto, enfatiza-se a importância de se alcançar esse resultado. ${ }^{12,13}$ 


\section{REFERÊNCIAS}

1. Llombart B, Serra-Guillén C, Monteagudo C, Guerrero JAL, Sanmartín O. Dermatofibrosarcoma protuberans: a comprehensive review and update on diagnosis and management. Semin Diagn Pathol. 2013;30:13-28.

2. Criscione VD, Weinstock MA. Descriptive epidemiology of dermatofibrosarcoma protuberans in the United States, 1973 to 2002. J Am Acad Dermatol. 2007; 56: 968-73.

3. Monnier D, Vidal C, Martin L, Danzon A, Pelletier F, Puzenat E, Algros MP, et al. Dermatofibrosarcoma protuberans: a population-based cancer registry descriptive study of 66 consecutive cases diagnosed between 1982 and 2002. J Eur Acad Dermatol Venereol. 2006; 20:1237-42.

4. McPeak CJ, Cruz T, Nicastri AD. Dermatofibrosarcoma protuberans: an analysis of 86 cases-five with metastasis. Ann Surg. 1967; 166:803-16.

5. Angouridakis N, Kafas P, Jerjes W, Triaridis S, Upile T, Karkavelas G, Nikolaou A. Dermatofibrosarcoma protuberans with fibrosarcomatous transformation of the head and neck. Head Neck Oncol. 2011; 3: 5.

6. Miller SJ, Alam M, Andersen JS, Berg D, Bichakjian CK, Bowen GM, et al. Dermatofibrosarcoma protuberans. JNCCN. 2012;10:312-18.

7. Chang C. K., Jacobs I. A., Salti G. I. Outcomes of surgery for dermatofibrosarcoma protuberans. Eur Surg Oncol. 2004;30:341-5.
8. Serra-Guillén C, Llombart B, Nagore E, Guillén C, Requena $C$, Traves $V$, et al. Mohs micrographic surgery in dermatofibrosarcoma protuberans allows tumour clearance with smaller margins and greater preservation of healthy tissue compared with conventional surgery: a study of 74 primary cases. $\mathrm{Br} J$ Dermatol. 2015; 172:1303-7.

9. McArthur G.A. Molecular targeting of dermatofibrosarcoma protuberans: a new approach to a surgical disease. J Natl Compr Canc Netw. 2007;5:557-62.

10. Bowne WB, Antonescu CR, Leung DH, Katz SC, Hawkins WG, Woodruff JM, et al. Dermatofibrosarcoma protuberans: a clinicopathologic analysis of patients treated and followed at a single institution. Cancer. 2000;88:2711-20.

11. Torreggiani WC, Al-Ismail K, Munk PL, Nicolaou S, O'Connell JX, Knowling MA. Dermatofibrosarcoma protuberans: MR imaging features. AJR Am J Roentgenol. 2002;178:989-93.

12. Hamid R, Hafeez A, Darzi AM, Zaroo I, Owais H, Akhter A. Dermatofibrosarcoma protuberans: role of wide local excision. South Asian J Cancer. 2013; 2: $232-8$

13. Love W. E., Keiler S. A., Tamburro J. E., Honda K., Gosain A. K., Bordeaux J. S. Surgical management of congenital dermatofibrosarcoma protuberans. J Am Acad Dermatol. 2009;61:1014-23. 